\title{
Extensions of lattice groups, gerbes and chiral fermions on a torus
}

\section{Mickelsson, Jouko}

2017-11

Mickelsson , J 2017 , ' Extensions of lattice groups, gerbes and chiral fermions on a torus ' , Journal of Geometry and Physics, vol. 121 , pp. 378-385 . https://doi.org/10.1016/j.geomphys.2017.08.003

http://hdl.handle.net/10138/304624

https://doi.org/10.1016/j.geomphys.2017.08.003

cc_by_nc_nd

acceptedVersion

Downloaded from Helda, University of Helsinki institutional repository.

This is an electronic reprint of the original article.

This reprint may differ from the original in pagination and typographic detail.

Please cite the original version. 
Geometry and Physics

Elsevier Editorial System(tm) for Journal of Manuscript Draft

Manuscript Number:

Title: Extensions of lattice groups, gerbes and chiral fermions on a torus

Article Type: VSI:String geometry, duality

Keywords: Extensions of current algebras; K theory; gerbes; topological insulators

Corresponding Author: Professor jouko mickelsson, Ph. D.

Corresponding Author's Institution: University of Helsinki

First Author: jouko mickelsson, Ph. D.

Order of Authors: jouko mickelsson, Ph. D.

Abstract: Motivated by the topological classification of hamiltonians in condensed matter

physics (topological insulators) we study the relations between chiral Dirac operators coupled to an abelian vector potential on a torus in 3 and 1 space dimensions.

We find that a large class of these hamiltonians in three dimensions is equivalent, in $K$ theory, to a family of hamiltonians in just one space dimension but with a different

abelian gauge group.

The moduli space of U(1) gauge connections over a torus with a fixed Chern class

is again a torus up to a homotopy. Gerbes over a n-torus can be realized in terms of extensions of

the lattice group acting in a real vector space. The extension comes from the action of the lattice group (thought of as "large" gauge

transformations,

homomorphisms from the torus to $\mathrm{U}(1)$ ) in the Fock space of chiral

fermions.

Interestingly, the $\mathrm{K}$ theoretic classification of Dirac operators coupled to vector

potentials in this setting in 3 dimensions can be related to families of Dirac operators on a circle with gauge group the 3-torus. 


\title{
田 \\ EXTENSIONS OF LATTICE GROUPS, GERBES AND CHIRAL FERMIONS ON A TORUS
}

\author{
JOUKO MICKELSSON
}

\begin{abstract}
Motivated by the topological classification of hamiltonians in condensed matter physics (topological insulators) we study the relations between chiral Dirac operators coupled to an abelian vector potential on a torus in 3 and 1 space dimensions. We find that a large class of these hamiltonians in three dimensions is equivalent, in $\mathrm{K}$ theory, to a family of hamiltonians in just one space dimension but with a different abelian gauge group.

The moduli space of $U(1)$ gauge connections over a torus with a fixed Chern class is again a torus up to a homotopy. Gerbes over a n-torus can be realized in terms of extensions of the lattice group acting in a real vector space. The extension comes from the action of the lattice group (thought of as "large" gauge transformations, homomorphisms from the torus to $\mathrm{U}(1)$ ) in the Fock space of chiral fermions. Interestingly, the $\mathrm{K}$ theoretic classification of Dirac operators coupled to vector potentials in this setting in 3 dimensions can be related to families of Dirac operators on a circle with gauge group the 3-torus.
\end{abstract}

\section{INTRODUCTION}

Topological classification of hamiltonians in condensed matter physics has attracted considerable interest in recent years. Families of hamilton operators parametrized by some topological space $X$ lead to a study of the $\mathrm{K}$ group $K(X)$ of $X$. Typically the physical space is compactified as a torus $T^{d}$ and the hamiltonians are some simple perturbations of the (chiral) Dirac operator on $T^{d}$. Often there are discrete symmetries (time reversal, charge conjugation, parity) which make the classification richer, in terms of the Real $\mathrm{K}$ theory groups $K R(X)$, giving the 'periodic table' for topological insulators [1], [2].

In this paper we shall just restrict us to the complex $\mathrm{K}$ theory groups $K(X)$. The Dirac operators are coupled to an abelian gauge potential (Maxwell field or its generalization). The real interest is in the case of space dimension $d=3$ but interestingly we can show that a large class of chiral hamiltonians is actually equivalent, in terms of $\mathrm{K}$ theory, to a family of hamiltonians on the circle $S^{1}$ with another abelian gauge group.

The canonical quantization of chiral hamilton operators leads to projective Hilbert bundle (a gerbe) over the moduli space of gauge connections. In the nonabelian case these have studied earlier in [6]. In the abelian case there are important simplifications. It turns out that the gerbe can be constructed from a central extension of a transformation groupoid, the lattice group $\mathbb{Z}^{3}$ acting on the real vector space $\mathbb{R}^{3}$.

A gerbe can be alternatively described in terms of local complex line bundles over intersections of open sets in a cover. In the case at hand, pulling back to $\mathbb{R}^{3}$ by the

\footnotetext{
${ }^{1}$ Invited talk at the conference "String Geometries and Dualities", IMPA, Rio de Janeiro, December 12 - 16, 2016
} 
projection $\pi: \mathbb{R}^{3} \rightarrow T^{3}$ the gerbe can be trivialized with respect to the pull-back of the open cover; this leads to a family of local complex line bundles over $\mathbb{R}^{3}$ with singularities at lattice points parametrized by the $\mathbb{Z}^{3}$ action. Interestingly, these line bundles are renormalized infinite sums of monopole line bundles at the lattice points, the total curvature coming from the curvature of a certain Grassmann manifold modelled by Schatten ideals. This construction can be viewed as a special case of the abstract gauge group extension in [12.

Finally, in Section 5 an alternative method for constructing gerbes over compact Lie groups is presented in terms of finite-dimensional groupoid extensions using a similar idea as in the construction of the central extension of the transformation groupoid $\mathbb{R}^{3} \times \mathbb{Z}^{3} \rightarrow \mathbb{R}^{3}$ through canonical quantization of fermions on a torus.

In the hamiltonian quantization of chiral fermions coupled to a nonabelian vector potential the group of gauge transformations acts through an abelian extension. In particular, in the case of 3-dimensional physical space $M$ the extension manifests as a 2-cocycle of the form [3, [4]

$$
c(X, Y)=\frac{i}{24 \pi^{2}} \int_{M} \operatorname{tr} A(d X d Y-d Y d X)
$$

for a pair $X, Y$ of infinitesimal gauge transformations acting on Weyl spinors and a gauge potential $A$, the trace being computed in a finite-dimensional representation of a compact gauge group $G$. The cocycle condition here reads

$c([X, Y], Z)+c([Y, Z], X)+c([Z, X], Y)-L_{X} c(Y, Z)-L_{Y} c(Z, X)-L_{Z}(X, Y) c=0$

with $L_{X}$ the Lie derivative acting on functions of the potential $A$. Ideally, the group $\mathcal{G}$ of smooth maps $M \rightarrow G$ would be represented in a Hilbert space arising from the quantization of fermions and the gauge connection. However, unlike in the case of a $1+1$ dimensional space time such a (faithful) representation is not known. The obstruction comes from the fact that no suitable measure is known to exist in the space $\mathcal{A}$ of vector potentials in three space dimensions.

In this paper we concentrate on the case of an abelian gauge field with gauge group $G=U(1)$. We assume that the 3 -dimensional space $M$ is compactified as the 3 -torus $T^{3}$. In this case the Lie algebra cocycle defining the extension becomes trivial, but there remains a global 2-cocycle supported in the group of large gauge transformations $\mathbb{Z}^{3}=\operatorname{Hom}\left(T^{3}, U(1)\right)$. It turns out that using the subgroup $\mathcal{G}_{0}$ of contractible gauge transformations the remaining degrees of freedom can be factorized as $\mathcal{A}^{\prime \prime} \oplus \mathbb{R}^{3}$ where also the first summand is contractible and the large gauge transformations $\mathbb{Z}^{3}$ act as translations on the space $\mathbb{R}^{3}$ of constant gauge connections on the torus.

The quantization of chiral fermions creates a 2-cocycle for the action of the group $\mathbb{Z}^{3}$. on $\mathbb{R}^{3}$. Since the residual gauge group action on the infinite dimensional part $\mathcal{A}$ is trivial we can concentrate on the action on the fermionic Fock spaces parametrized by the constant potentials $\mathbb{R}^{3}$. Using the Lebesgue measure on $\mathbb{R}^{3}$ we can define the Hilbert space $\mathcal{H}$ as the space of square integrable functions on $\mathbb{R}^{3}$ with values in the fermionic Fock space $\mathcal{F}$. The extension of the gauge group $\mathbb{Z}^{3}$ is then unitarily and faithfully represented in $\mathcal{H}$.

This paper was inspired by several discussions on condensed matter problems and Schwinger terms with Edwin Langmann, which is gratefully acknowledged. 


\section{Topological Classification of the Dirac operators on a 3-TORUS}

Let us specify the setting for 1-particle fermions. We fix a compact 3-dimensional spin manifold $M$, later to be fixed the 3 -torus $T^{3}$. Normally, the abelian extension of a gauge current algebra comes from coupling a nonabelian vector potential $A$ (1-form on $M$ with values in the Lie algebra $\mathfrak{g}$ of a compact gauge group $G$ ) to 2-component Weyl fermions. The canonical quantization of the Weyl fermions induces the 2-cocycle (1.1) on the Lie algebra $\operatorname{Map}(M, \mathfrak{g})$ when acting in the fermionic Fock spaces coupled to $A$.

Let us now change the setting in the following way. Consider Dirac fermions (4 components) coupled to the nonabelian vector potential $A$ in the usual way, and in addition let us couple the fermions chirally to an abelian vector potential $a$; that is, $a$ is only coupled to the left handed fermions but not to the right handed fermions.

The chiral anomaly is computed using the families index theorem [5]. In four space-time dimensions one takes the 6-form part of the index form

$$
\operatorname{tr} \hat{A} e^{F / 2 \pi i}
$$

where $\hat{A}$ is the A-roof genus computed from the Riemann tensor and $F$ is the curvature form $F=d A+\frac{1}{2}[A, A]$. In most cases in 4 dimensions $\hat{A}$ vanishes (and in particular on a torus or a sphere) so let us assume that this is the case. The 6 -form part is then

$$
\frac{1}{6 \cdot(2 \pi i)^{3}} \operatorname{tr} F^{3} \text {. }
$$

The chiral anomaly and the Schwinger terms are calculated by transgression starting from the above expression. However, in the case of Dirac fermions the contributions from the left and right sectors come with opposite signs and they cancel. But now is $a$ is chirally coupled to the fermions there is a piece which is left over, namely

$$
\frac{i}{24 \pi^{2}} \operatorname{tr}\left(f^{3}+3 f F^{2}\right) .
$$

Here $f=d a$ is the field strength of the abelian (Maxwell) potential. Again by transgression this leads to a mixed Schwinger term

$$
c(X, Y)=\frac{i}{24 \pi^{2}} \int_{M} f \operatorname{tr}(X d Y-Y d X) .
$$

Here $f$ is fixed as the field strength of the external Maxwell field. Thus we get a central extension of the current algebra $\operatorname{Map}(M, \mathfrak{g})$.

The above central extension has an operator theoretic derivation similar to the construction of the current algebra in $1+1$ dimensions in the fermionic Fock space. Now the grading operator is the sign $\epsilon_{a}$ of the hamiltonian $D_{a}=D_{0}+\frac{1}{2}\left(\gamma_{5}+1\right) a$ where $D_{0}$ is the free Dirac hamiltonian.

Then one can check that $\left[\epsilon_{a}, X\right]$ is conditionally Hilbert-Schmidt. Conditionally means here that when computing traces of operators one has to take first the trace over spin and gauge algebra indices and then perform space and momentum integration for pseudodifferential operators. The trace over spin indices for Dirac fermions makes that the diverging contributions from the left and right sector cancel. They cancel totally if $a=0$ but in the case of $a \neq 0$ there is a left-over piece when expanding $\epsilon_{a}$ in powers of $a$. This calculation can be done explicitly 
using residue calculus [7, [8]. In the canonical quantization of fermions in $1+$ 1 dimensions the gauge algebra is centrally extended and the 2-cocycle of the extension can be evaluated (when the physical space is compactified as a circle $S^{1}$ ) from

$$
\frac{1}{2} \operatorname{tr}_{C} X[\epsilon, Y]=\operatorname{Res} \epsilon X[\ell, Y]=\frac{1}{2 \pi i} \int_{S^{1}} \operatorname{tr} X d Y
$$

for a pair $X, Y: S^{1} \rightarrow \mathfrak{g}$ of infinitesimal gauge transformations where $\ell$ is a logarithmic symbol (the log of the momentum operator in one dimension) and Res is the Wodzicki operator residue. Here the conditional trace $\operatorname{tr}_{C}$ is calculated in a bases where $\epsilon$ is diagonal. In general, the first equation is true only up to coboundaries of cocycles but it can always be used when the Hilbert-Schmidt condition is satisfied for the off-diagonal blocks.

Now let us concentrate on the abelian case with gauge group $U(1), A=0$ and $X, Y: M \rightarrow i \mathbb{R}$ infinitesimal gauge transformations acting on the abelian vector potential $a$. We also assume that the physical space $M$ is the 3 -torus $T^{3}$. At the first sight the extension of the gauge algebra defined by the cocycle $c$ is trivial since $c=\delta b$ where $b$ is the cochain

$$
b(a ; X)=\frac{-i}{24 \pi^{2}} \int_{T^{3}} d a \wedge a X .
$$

However, this is not the whole story. First, the group $\mathcal{G}$ of gauge transformations $g: T^{3} \rightarrow U(1)$ is disconnected,

$$
\mathcal{G}=\mathbb{Z}^{3} \times \mathcal{G}_{0}
$$

where the elements of $\mathbb{Z}^{3}$ corresponds to the large gauge transformations $g\left(x_{1}, x_{2}, x_{3}\right)=$ $\exp 2 \pi i \mathbf{n} \cdot \mathbf{x}$ with $\mathbf{n} \in \mathbb{Z}^{3}$ and $\mathcal{G}_{0}$ consists of the contractible maps $T^{3} \rightarrow U(1)$ which can be written as $g=\exp 2 \pi X$ with $X: T^{3} \rightarrow i \mathbb{R}$ periodic on the interval $[0,1]^{3}$.

We can define a group cocycle on $\mathcal{G}$ such that the corresponding Lie algebra cocycle is $c$. It is given by the formula

$$
C\left(a ; g, g^{\prime}\right)=e^{2 \pi i \int_{T^{3}} a \wedge d X \wedge d Y}
$$

with $g^{\prime}=\exp 2 \pi Y$. In particular, when restricted to $\mathbb{Z}^{3}$ the cocycle takes the form

$$
C(a ; \mathbf{n}, \mathbf{m})=e^{2 \pi i \mathbf{a} \wedge \mathbf{n} \wedge \mathbf{m}}
$$

for constant potentials $\mathbf{a}=a_{1} d x_{1}+a_{2} d x_{2}+a_{3} d x_{3}$. This gives all topological information about the cocycle since $\mathcal{A} / \mathcal{G}_{0}$ is homotopic to the space of constant vector potentials modulo the group $\pi_{1}\left(T^{3}\right)=\mathbb{Z}^{3}$ of large gauge transformations, i.e., the homomorphisms from $T^{3}$ to $S^{1}$. This follows from the fact that any potential $a$ on the torus $T^{3}$ is gauge equivalent to a potential $A^{\prime}$ with $\sum \partial_{i} A_{i}^{\prime}=0$, through a contractible gauge transformation. The space of divergence free potentials is a direct sum of the space of constant potentials and its orthogonal complement, the space $\mathcal{A}^{\prime \prime}$ of those divergence free potentials $A$ with

$$
\int_{T^{3}} a_{i} d^{3} x=0
$$

for $i=1,2,3$. The large gauge transformations act on $\mathcal{A}^{\prime \prime} \oplus \mathbb{R}^{3}$ as translations by $\mathbb{Z}^{3}$ on the second summand. 
Let $\mathcal{G}_{b}$ be the group of based gauge transformations $g$, i.e., $g(0,0,0)=1$. This group acts freely on $\mathcal{A}$ and from the above discussion follows that up to homotopy the moduli space $\mathcal{A} / \mathcal{G}_{b}$ can be identified as the set of constant potentials, parametrized by $\mathbb{R}^{3}$, modulo the action of $\mathbb{Z}^{3}$, i.e., $\mathcal{A} / \mathcal{G}_{b}=T_{a}^{3}$. The moduli space torus is denoted by $T_{a}^{3}$ whereas $T_{x}^{3}$ will denote the physical space.

Coming back to the case of Dirac operators on the torus $T^{3}$ coupled to abelian gauge potentials $a$ we again restrict to the constant potentials since the moduli space is $\mathcal{A} / \mathcal{G}_{b} \simeq \mathbb{R}^{3} / \mathbb{Z}^{3}=T_{a}^{3}$. When $a$ is variable, the potential $a=\sum_{i} a_{i} d x_{i}$ is a potential on $\mathbb{R}_{a}^{3} \times T_{x}^{3}$; it is only locally defined on $T_{a}^{3} \times T_{x}^{3}$ but its curvature $F=\sum_{i} d a_{i} \wedge d x_{i}$ descends to $T_{a}^{3} \times T_{x}^{3}$. With the families index formula this gives the 3 -form

$$
\Omega=\frac{1}{(2 \pi i)^{3}} \int_{T_{x}^{3}} F^{3}=d a_{1} \wedge d a_{2} \wedge d a_{3}
$$

on the moduli torus $T_{a}^{3}$.

We have assumed that the vector potential $a$ is globally defined, i.e., it comes from complex line bundle over $T_{x}^{3}$ with vanishing Chern class. In the case of non vanishing Chern class we can write a connection in the form $\nabla+a$ where $\nabla$ is a fixed connection and $a$ is globally defined. One can then repeat the above considerations in this case. The cohomology $\mathrm{H}^{2}\left(T^{3}, \mathbb{Z}\right)$ is isomorphic to $\mathbb{Z}^{3}$ so the total moduli space for all complex line bundles over $T_{x}^{3}$ becomes $\mathbb{Z}^{3} \times T_{a}^{3}$.

The form $\Omega$ is the Dixmier-Douady class of a complex projective vector bundle. In the canonical quantization of fermions chirally coupled to a vector potential the bundle of Fock spaces is defined over the covering $\mathbb{R}_{a}^{3}$ but there is an obstruction coming from a nonzero $\Omega$ to push it to a bundle over the moduli space $T_{a}^{3}$. Alternatively, the obstruction is described by the central extension of the $\mathbb{Z}^{3}$ action on $\mathbb{R}_{a}^{3}$ given by (2.3).

Let $S_{i j k}$ with $i, j, k=1,2,3$ be any tensor with integer entries. Then

$$
C(a ; n, m)=e^{2 \pi i \sum S_{j k l} a_{j} n_{k} m_{l}}
$$

defines an extension for the $\mathbb{Z}^{3}$ action on $\mathbb{R}^{3}$. However, the Dixmier-Douady class corresponding to this extension is

$$
\sum S_{i j k} d a_{i} \wedge d a_{j} \wedge d a_{k}
$$

and therefore depends only on the antisymmetrization $S$ which is $p \cdot \epsilon_{i j k}$ where $p \in \mathbb{Z}$ and $\epsilon$ is the unique totally antisymmetric tensor with $\epsilon_{123}=1$, [10], Section 7. The integer $p$ is equal to $\frac{1}{6} \sum \epsilon_{i j k} S_{i j k}$.

The Dixmier-Douady class is the only topological invariant of a projective complex Hilbert bundle. However, the families index theorem gives characteristic classes in any odd dimension for a family of self adjoint Fredholm operators. In our case the parameter space is $T^{3}$ so the odd cohomology is nonvanishing only in dimensions 1 and 3 . The element in $\mathrm{H}^{1}\left(T^{3}, \mathbb{Z}\right)$ describes the spectral flow for a family of hamiltonians. It is again computed from the index theorem by taking the form $F^{2}$ on $T_{x}^{3} \times T_{a}^{3}$ and integrating over $T_{x}^{3}$. For a trivial complex line bundle over $T_{x}^{3}$ this gives zero since the $(3,1)$ component of $F^{2}$ vanishes. However, twisting with a non trivial line bundle with curvature $f=f_{1} d x_{2} \wedge d x_{2}+f_{2} d x_{3} \wedge d x_{1}+f_{3} d x_{1} \wedge d x_{2}$ the $(3,1)$ component becomes $f \wedge \sum_{i} d a_{i} \wedge d x_{i}$ and its integral over $T_{x}^{3}$ is equal to the 1 -form $\sum_{i} f_{i} d a_{i}$ on $T_{a}^{3}$. 
Next we show that up to homotopy the same class of projective Dirac operators over the parameter space $T_{a}^{3}$ is obtained 1D Dirac operators on a unit circle $S_{\theta}^{1}$. One can apply the construction in [11, Section 2. Let us write $T^{3}$ as the product $S_{\phi}^{1} \times M$ with $M=T^{2}$. Fix a an element $\beta \in \mathrm{H}^{2}(M, \mathbb{Z}) \simeq \mathbb{Z}$. Now $\mathrm{H}^{1}\left(S^{1}, \mathbb{Z}\right) \simeq \mathbb{Z}$ and we choose the angular form $d \phi$ as the generator; the circle $S_{\phi}^{1}$ is a parameter, in addition to $M$, for a family of Dirac operators whereas the 1D Dirac operator is defined on the circle $S_{\theta}^{1}$. The parameter $\phi$ measures the holonomy around $S_{\theta}^{1}$ of the Dirac operator coupled to a constant vector potential.

The family of Dirac operators is then twisted with a connection on $S_{\theta}^{1} \times S_{\phi}^{1} \times M$ with total curvature $F=\frac{i}{2 \pi} d \theta \wedge d \phi+\beta$ and the index form on $S_{\phi}^{1} \times M$ becomes

$$
\int_{S_{\theta}^{1}} e^{F / 2 \pi i}=\frac{d \phi}{2 \pi}+\frac{d \phi}{2 \pi} \wedge \frac{\beta}{2 \pi i} .
$$

In particular, $\beta \mapsto d \phi \wedge \beta$ gives an isomorphism between $\mathrm{H}^{2}(M, \mathbb{Z})$ and $\mathrm{H}^{3}\left(T^{3}, \mathbb{Z}\right)$.

The above construction can be slightly generalized noting that we have arbitrarily chosen one circle $S_{\phi}^{1}$ in $T^{3}$. We can also take direct sums of 1D Dirac operators corresponding to three different choices of the parameter circle $S_{\phi}^{1}$ inside $T^{3}$. Giving weights $\left(f_{1}, f_{2}, f_{3}\right) \in \mathbb{Z}^{3}$ to the different choices leads to the index form $\sum_{i} f_{i} d x_{i}$ in $\mathrm{H}^{1}\left(T^{3}, \mathbb{Z}\right)$ and to the Dixmier-Douady class $f \wedge \beta / 2 \pi i$ in $\mathrm{H}^{3}\left(T^{3}, \mathbb{Z}\right)$ for a given line bundle on $T^{3}$ with curvature $\beta$. More concretely, we may consider a family of Dirac operators on the unit circle $S_{\theta}$ coupled to an abelian gauge connection with the structure group $T^{3}$. Then the moduli space of gauge connections is again $T^{3}$ and we may twist the family of Dirac operators by a complex line bundle over $S_{\theta}^{1} \times T^{3}$ with curvature $f=d \theta \wedge \alpha+\beta$ where $\alpha / 2 \pi i$ is an integral 1-form on $T^{3}$ and $\beta / 2 \pi i$ is an integral 2 -form on $T^{3}$. Then

$$
\int_{S_{\theta}^{1}} e^{f / 2 \pi i}=\alpha / 2 \pi i+\alpha \wedge \beta /(2 \pi i)^{2} .
$$

In conclusion, we have

Theorem 2.1. The odd K-theory classes on $T^{3}$ generated by Dirac operators $D_{A}$ coupled to $U(1)$ gauge connection $A$ on the torus can be alternatively defined by 1 dimensional Dirac operators in the fibers of a circle bundle over a 3-torus provided that the greatest common divisor of the components $\beta_{i j}$ is equal to one.

Proof. The Chern character map from $K^{*}\left(T^{3}\right)$ to $\mathrm{H}^{*}\left(T^{3}, \mathbb{Z}\right)$ is an isomorphism. The Chern character in the case of the 3D Dirac operators described above is the generator of $\mathrm{H}^{3}\left(T^{3}, \mathbb{Z}^{3}\right)$ together with the degree one component $f \in \mathrm{H}^{1}\left(T^{3}, \mathbb{Z}\right)$. In the case of the family of $1 \mathrm{D}$ Dirac operators the Chern character is $f$ in degree one and $\sum \beta_{i} f_{i}$ times the basic form in degree three. The latter has the value 1 for a suitable $\beta$ if the greatest common divisor of the components $f_{i}$ is one.

\section{QuAntization of 1D FERMions With an ABelian gaUge group}

As we have seen, up to homotopy, the moduli space of $U(1)^{3}$ gauge connections on the unit circle $S^{1}$ is a three torus $\mathbb{R}^{3} / \mathbb{Z}^{3}=T_{a}^{3}$ where the group $\mathbb{Z}^{3}$ consists of the gauge transformations $\left(e^{i n_{1} x}, e^{i n_{2} x}, e^{i n_{3} x}\right)$ with $0 \leq x \leq 2 \pi$ acting on the constant potentials $a$ as $a^{i} \mapsto a^{i}+n_{i}$. Fix an element $\Omega \in \mathrm{H}^{3}\left(T_{a}^{3}, \mathbb{Z}\right)$. We construct 
a projective bundle of fermionic Fock spaces over $T_{a}^{3}$ corresponding to the DixmierDouady class $\Omega$.

To start with consider the trivial bundle $\mathbb{R}^{3} \times \mathcal{F}$ over $\mathbb{R}^{3}$ where $\mathcal{F}$ carries a representation of the canonical commutation relations algebra (CAR) with a vacuum vector $\mid 0>$. The CAR algebra is generated by the elements $b^{*}(v)$ and $b(v)$ with $v \in H=L^{2}\left(S^{1}, \mathbb{C}^{3}\right)$ with the nonzero anticommutators

$$
b^{*}(u) b(v)+b(v) b^{*}(u)=<u, v>_{L^{2}} \cdot \mathbf{1}
$$

when $u, v$ are proportional to the same basis vector $e_{i} \in \mathbb{C}^{3}$. We require that the fermions corresponding to the 3 different coordinates in $\mathbb{C}^{3}$ commute with each other; this is not essential, we could make them anticommute, this is only to make certain sign conventions later on simpler. The vacuum vector is characterized by the property

$$
b^{*}(u)|0>=0=b(v)| 0>
$$

for $u \in H_{-}$and $v \in H_{+}$where $H_{-}$(resp. $H_{+}$) is the subspace spanned by the nonpositive (resp. positive) Fourier modes on the circle $S^{1}$.

Let $v_{j, p}(x)=\frac{1}{\sqrt{2 \pi}} e^{i p x} e_{j}$ denote the Fourier modes in the $j:$ th direction $e_{j} \in \mathbb{C}^{3}$. For $n \in \mathbb{Z}^{3}$ set $b^{*}(n)=b^{*}\left(\sum_{j} v_{j, n_{j}}\right)$ and likewise for $b(n)$. Next we twist these modes by a 1-cocycle over $\mathbb{R}^{3}$ allowing the operators depend on $a \in \mathbb{R}^{3}$ such that

$$
b^{*}(n, a+m)=e^{i a \wedge \beta \wedge m} b^{*}(n, a)
$$

where $\beta \in \mathbb{Z}^{3}$ is a fixed vector. This means that the fermion operators are twisted by a complex line bundle over $T^{3}$ with curvature labelled by the components of the vector $\beta$.

The action of the gauge transformations $g(m)$ in the Fock space is now completely fixed by the condition

$$
g(m) b^{*}(n, a) g(m)^{-1}=b^{*}(n+m, a+m)=e^{i a \wedge \beta \wedge m} b^{*}(n+m, a)
$$

and the action on the vacuum vector

$$
g(m)\left|0>=\left(\prod_{i} S_{i}^{\alpha_{i} m_{i}}\right)\right| 0>.
$$

Here $S_{i}$ is the shift operator in the $i$-direction, increasing the fermion number by one unit;

$$
\left.S_{i} \mid 0>=b^{*}\left(v_{i, 1}\right)\right) \mid 0>.
$$

The vector $\alpha \in \mathbb{Z}^{3}$ corresponds to the spectral flow 1-form in the previous section. We have $S_{i} N_{j} S_{i}^{-1}=N_{j}+\delta_{i j}$ where

$$
N_{j}=\sum_{p}: b^{*}\left(v_{j, p}\right) b\left(v_{j, p}\right):=\sum_{p>0} b^{*}\left(v_{j, p}\right) b\left(v_{j, p}\right)-\sum_{p \leq 0} b\left(v_{j, p}\right) b^{*}\left(v_{j, p}\right) .
$$

The shift operator $S_{i}$ is the quantization of the 1-particle space shift operator $s_{i} v_{j, p}=v_{j, p+\delta_{j, i}}$ in the i direction in $L^{2}\left(S^{1}, \mathbb{C}^{3}\right)$.

Now we can compute the twisted action of $\mathbb{Z}^{3}$ in $\mathcal{F}$. Denote by $\mid N>$ the subspace with fermion numbers $N=\left(N_{1}, N_{2}, N_{3}\right)$. For a given $k \in \mathbb{Z}$ we get

$$
g(n)\left|N>=e^{2 \pi i k a \cdot n \wedge N}\left(\prod S_{i}^{n_{i}}\right)\right| N>.
$$

Then we observe that

$$
g(n) g(m)=e^{2 \pi i(\alpha \cdot m) a \wedge n \wedge \beta} g(n+m)=C(a ; n, m) g(n+m) .
$$


Up to a coboundary, this can be written in the antisymmetric form

$$
C(a ; n, m)=e^{2 \pi i k a \wedge n \wedge m}
$$

where $k=\alpha \cdot \beta$. Thus $\mathbb{Z}^{3}$ is acting through an abelian extension defined by the 2-cocycle $C$ with values in the abelian group of exponential $S^{1}$ valued functions on $\mathbb{R}^{3}$. This extension is actually central since $C(a+p ; n, m)=C(a ; n, m)$ for all $p \in \mathbb{Z}^{3}$. The integer $k$ corresponds to the Dixmier-Douady class $\Omega \in \mathrm{H}^{3}\left(T^{3}, \mathbb{Z}\right)=\mathbb{Z}$.

The quantized Dirac operator coupled to the constant vector potential $a$ can now be written as

$$
\hat{D}_{a}=\sum_{j, p} j_{p}: b^{*}\left(v_{j, p}\right) b\left(v_{j, p}\right):-\sum_{i=1}^{3} a_{i} J_{0}^{i}+\frac{1}{2} \sum_{i=1}^{3} a_{i}^{2}
$$

where $J_{0}^{i}$ is the zero Fourier component of the gauge current in the $i$ direction,

$$
J_{0}^{i}=\sum_{p}: b^{*}\left(v_{i, p}\right) b\left(v_{i, p}\right):+\frac{1}{2}=N_{i}+\frac{1}{2}
$$

where again the $v_{i, p}$ 's are the Fourier modes in the $i$ direction in $\mathbb{C}^{3}$. One can check by a direct computation that indeed the family of hamiltonians transforms covariantly under the gauge transformations, $g(n)^{-1} \hat{D}_{a} g(n)=\hat{D}_{a+n}$.

There is an even simpler realization of the gerbe as a projective vector bundle (but not of the family of quantized Dirac operators) corresponding to the same Dixmier-Douady class defined by the groupoid cocycle $c$ above. Let $H$ be a complex Hilbert space with an orthonormal basis labelled by integers $N$. Fix a pair of vectors $p, q \in \mathbb{Z}^{3}$. Let $\mathcal{H}=L^{2}\left(\mathbb{R}^{3}, H\right)$ and for $\psi \in \mathcal{H}$ set

$$
(g(n) \psi)_{N}(a)=e^{2 \pi i N a \wedge p \wedge n} \psi(a-n)_{N-q \cdot n}
$$

where $\psi_{N}(a)$ denotes the component of the vector $\psi(a)$ in the given basis. The 2-cocycle computed from the action is

$$
C^{\prime}(a ; n, m)=e^{-2 \pi i(q \cdot m) a \wedge p \wedge n} .
$$

It differs from $C$ by a coboundary for $k=p \cdot q$ : The equivalence classes of $S^{1}$ 2-cocycles for the transformation groupoid $\mathbb{R}^{3} \times \mathbb{Z}^{3} \rightarrow \mathbb{R}^{3}$ correspond to equivalence classes of gerbes over $T^{3}$ which are classified by $\mathrm{H}^{3}\left(T^{3}, \mathbb{Z}\right)=\mathbb{Z}$. The antisymmetrization of $C^{\prime}$ is exactly $C$ when $k=p \cdot q$. The Dixmier-Douady class of the gerbe is obtained from $\frac{1}{2 \pi i} \delta \log C$ which generates the group cohomology $\mathrm{H}_{\text {grp }}^{3}\left(\mathbb{Z}^{3}, \mathbb{Z}\right)=\mathbb{Z}=\mathrm{H}^{3}\left(T^{3}, \mathbb{Z}\right),[10]$, Section 7 .

\section{Quantization of 3D fermions with gauge group $U(1)$}

In 3 space dimensions there is a technical problem related to the fact that only constant gauge transformations can be canonically implemented in the fermionic Fock space, [13]. However, one can circumvent this obstacle as follows, [7]. For any smooth vector potential $a$ one constructs an unitary operator $T_{a}$ in the 1-particle space such that

$$
T(a, g)=T_{a^{g}}^{-1} g T_{a}
$$

has the property that $[\epsilon, T(a, g)]$ is Hilbert-Schmidt for a smooth gauge transformation $g$. This method can be applied also in the nonabelian case. 
Now $T(a, g)$ can be canonically quantized in the fermionic Fock space; the quantization is uniquely defined up to a complex phase. In our case, restricting to the constant vector potentials, the gauge transformation are simply shifts $a \mapsto a+n$ for $n \in \mathbb{Z}^{3}$. Denote the quantized operators as $g(n)$. Now $g(n)$ acts in the free fermionic Fock space. But because of the nontrivial Dixmier-Douady class in $\mathrm{H}^{3}\left(T^{3}, \mathbb{Z}\right)$ computed by the index theory argument before, the action is projective,

$$
g(n) g(m)=C(a ; n, m) g(n+m) .
$$

The structure of the gerbe over $T^{3}$ coming from the fermionic quantization can be further analyzed in a very concrete manner. Let $\lambda \in \mathbb{R}$ and define $U_{\lambda}=\{a \in$ $\left.\mathcal{A} \mid \lambda \notin \operatorname{Spec}\left(D_{a}\right)\right\}$. Then $U_{0}=\left\{a \in \mathbb{R}^{3} \backslash \mathbb{Z}^{3}\right\}$ and $\mathbb{R}^{3}=U_{0} \cup U_{1 / 3}$. The complex line bundle of fermionic vacua over $U_{0}$ has curvature

$$
\omega=\frac{1}{4} \operatorname{tr}_{C}(F-\epsilon) d F d F
$$

where $F=D_{a} /\left|D_{a}\right|$ and $\epsilon=D_{0} /\left|D_{0}\right|$ (in the subspace of zero modes, i.e. 3momentum equal to zero, we fix the action of $\epsilon$ as multiplication by zero) and the subscript $C$ refers to the conditional $\operatorname{trace} \operatorname{tr}_{C}(X)=\frac{1}{2} \operatorname{tr}(X+\epsilon X \epsilon)$. In the following we write the discrete momentum as a complex hermitean $2 \times 2$ matrix $p=\sum_{i} p_{i} \sigma_{i}$ with $\sigma_{i}$ the triple of Pauli matrices and similarly for the potential $a$. This means that in the momentum basis

$$
\omega=\frac{1}{8} \sum_{p \in \mathbb{Z}^{3}} \operatorname{tr}\left(\frac{p+a}{|p+a|}-\frac{p}{|p|}\right) d \frac{p+a}{|p+a|} \wedge d \frac{p+a}{|p+a|}
$$

where again $p /|p|$ is set to zero when $p=0$ and the trace here is the $2 \times 2$ matrix trace.

In a similar way the curvature of the vacuum line bundle over $U_{1 / 3}$ is evaluated by replacing $D+a$ by $D+a-\frac{1}{3}$.

In general, a gerbe is a projective Hilbert bundle over some space $X$. It can described alternatively in terms of local complex line bundles $L_{\alpha \beta}$ over intersections $V_{\alpha} \cap V_{\beta}$ of elements $V_{\alpha}$ of an open cover of $X$ with prescribed isomorphims $L_{\alpha \beta} \otimes$ $L_{\beta \gamma} \equiv L_{\alpha \gamma}$ leading to the $S^{1}$ valued cocycle $f_{\alpha \beta \gamma} \cdot \mathbf{1}=L_{\alpha \beta} \otimes L_{\beta \gamma} \otimes L_{\gamma \alpha}$. Then if $\pi: Y \rightarrow X$ is a projection and $Y$ is contractible we may write

$$
\pi^{*}\left(L_{\alpha \beta}\right)=L_{\alpha} \otimes L_{\beta}^{-1}
$$

for a family of local line bundles $L_{\alpha} \rightarrow U_{\alpha}=\pi^{-1}\left(V_{\alpha}\right)$ over $Y$. This is exactly the case above, with $Y=\mathbb{R}^{3}$ and $X=T^{3}$.

The curvature is formally a sum of two terms: The first is tr $F d F d F$ which is an extension of the curvature formula on a finite-dimensional Grassmannian to the infinite dimensional setting and the second is the exact term $\operatorname{tr} \epsilon d F d F$. Both diverge separately when one computes the infinite sum over momenta $p$. However, for a fixed momentum $p$ the first term gives

$$
\omega^{(1)}(p)=\frac{\sqrt{-1}}{4} \sum_{i j k} \epsilon_{i j k} \frac{(a+p)_{i} d a_{j} \wedge d a_{k}}{|p+a|^{3}}
$$

for $a \in \mathbb{R}^{3}$. This is the curvature of a unit magnetic monopole located at the point $-p$, with period $2 \pi \sqrt{-1}$. So the total curvature is the sum of curvatures of 
magnetic monopoles located in the infinite lattice $\mathbb{Z}^{3} \subset \mathbb{R}^{3}$ but renormalized by the subtraction of the infinite sum of exact forms located at the same lattice points.

Let us also briefly consider the case of Dirac hamiltonians in the even dimensional case, $d=4$, in the simple situation when the first Chern class of the $U(1)$ gauge field over the torus $T_{x}^{4}$ is zero. So again all the topological information is in the holonomies around the four different circles in $T_{x}^{4}$ which form the group $T_{a}^{4}$. Now the Dirac spinors have 4 complex components and we have the chirality operator $\Gamma$ with $\Gamma^{2}=1$, anticommuting with the Dirac operators.

The families index theorem gives now even characteristic classes on the moduli space $T_{a}^{4}$ of $U(1)$ gauge potentials. The cohomology in dimension 4 is one dimensional and what corresponds to the gerbe form $\Omega$ before is now the generator in $\mathrm{H}^{4}\left(T^{4}, \mathbb{Z}\right)=\mathbb{Z}$. The local trivialization on $\mathbb{R}^{4}$ are now local closed 3 -forms.

As before, the Dirac operator is invertible when $a \in \mathbb{R}^{4} \backslash \mathbb{Z}^{4}$. On this set of potentials the 3 -form

$$
\omega_{3}=\operatorname{tr}_{C} \Gamma(F-\epsilon) d F \wedge d F \wedge d F
$$

is well-defined. We can compute it at each momentum vector $p \in \mathbb{R}^{4}$ and the result is

$$
\omega_{3}^{(1)}(p)=\frac{(a+p) \wedge d a \wedge d a \wedge d a}{|p+a|^{4}}
$$

for the first term; the renormalization term involving the operator $\epsilon$ is the exact form

$$
\omega_{3}^{(2)}(p)=d \operatorname{tr} \Gamma \epsilon \frac{p+a}{|p+a|} d \frac{p+a}{|p+a|} \wedge d \frac{p+a}{|p+a|} .
$$

Summing over $p$, both $\omega_{3}^{(1)}(p)$ and $\omega_{3}^{(2)}(p)$ diverge but their difference is convergent.

\section{Gerbes on compact simple Lie groups}

Gerbes over compact simple Lie groups have been constructed in several ways; using the quantization of chiral fermions see [6], or more direct constructions [14, [16], [15]. As an application of the ideas in the previous sections we give another construction using finite dimensional groupoid extensions.

Let $G$ be a simple simply connected compact Lie group. The third cohomology $\mathrm{H}^{3}(G, \mathbb{Z})$ is isomorphic to the group $\mathbb{Z}$. Let us fix a de Rham representative $\Omega$ of a class of level $k \in \mathbb{Z}$. Let $\tilde{\Omega}$ be the pull-back of $\Omega$ with respect to the exponential mapping $\exp : \mathfrak{g} \rightarrow G$. We can then choose a 2 -form $\theta$ on $\mathfrak{g}$ such that $d \theta=\tilde{\Omega}$. The form $\theta$ gives in a natural way a closed 2-form on a groupoid $E X P$; this follows from the fact that $\Omega$ vanishes along surfaces in $\mathfrak{g}$ mapped to a constant by the exponential function. The closed form defines a circle extension $E \tilde{X} P$ of $E X P$. The sources and targets of $E X P$ are points $s, t$ in $\mathfrak{g}$ such that $e^{s}=e^{t}$ and the corresponding arrow is the vector $t-s$. The set of arrows starting from a point $s$ is disconnected. For example, when $s=0$ the set $T_{0}$ of targets are the points $t$ such that $e^{t}=1$. Restricted to the Cartan subalgebra $\mathfrak{h} \subset \mathfrak{g}$ these points are the points in the integral lattice $L$ generated by $2 \pi$ times the coroots $h_{\alpha} \in \mathfrak{h}$ and $\mathfrak{h} / L$ is the maximal torus $H \subset G$. The set $T_{0}$ consists then from the set of $G$ adjoint orbits through $L$. In the generic case the adjoint orbit through a point $t \in L$ is the smooth surface $G / H$.

By the homotopy exact sequence $G / H$ is simply connected, $\pi_{2}(G / H)=\pi_{1}(H)=$ $\mathbb{Z}^{\ell}$ with $\ell=\operatorname{rank}$ of $G$. It follows that also $\mathrm{H}_{2}(G / H, \mathbb{Z})=\mathbb{Z}^{\ell}$. The homology basis 
in $\mathrm{H}_{2}(G / H, \mathbb{Z})$ is given by the 2-spheres $S_{\alpha}^{2}$ of $S U_{\alpha}(2)$ orbits through $2 \pi h_{\alpha}$ where $S U_{\alpha}(2)$ is the subgroup with Lie algebra $\mathfrak{g}_{\alpha}$ corresponding to the simple root vectors $e_{ \pm \alpha}$ and the coroot vector $h_{\alpha}$. The image of these spheres in $\mathfrak{g}$ is the unit in $G$ in the exponential mapping.

The level $k$ on $G$ is of the form $\Omega$ is

$$
\Omega=\frac{k}{24 \pi^{2}}<d g g^{-1}, \frac{1}{2}\left[d g g^{-1}, d g g^{-1}\right]>
$$

where the invariant form $\langle\cdot, \cdot\rangle$ is normalized such that the length squared of the longest root becomes 2. The form $\theta$ can be written as [9]

$$
\theta_{Z}(X, Y)=\frac{k}{4 \pi^{2}}<X, h\left(a d_{Z}\right) Y>
$$

for tangent vectors $X, Y$ at a point $Z \in \mathfrak{g}$, with

$$
h(z)=\frac{\sinh (z)-z}{z^{2}} .
$$

To check the normalization of the 2 -form $\theta$ we just need to pair it against the homology cycles $S_{\alpha}^{2}$. But for $Z=2 \pi h_{\alpha}$ the form $\theta_{Z}$ gives $k / 16 \pi^{2}$ the area form on $S_{\alpha}^{2}$ at the point $Z$. On the other hand, the radius squared of $S_{\alpha}^{2}$ is $\|Z\|^{2}=$ $\left\|2 \pi h_{\alpha}\right\|^{2}=8 \pi^{2}$ so the integral becomes $4 \pi \cdot 8 k \pi^{2} / 16 \pi^{3}=2 k$. This is what one should expect since the ball in $\mathfrak{g}_{\alpha}$ with boundary $S_{\alpha}^{2}$ covers $S U_{\alpha}(2)$ twice in the exponential mapping.

The circle extension of $E X P$ given by the closed form $\theta$ fixes uniquely the class of the gerbe defined by the 3 -form $\Omega$. The 3 -homology of $G$ is generated by any of the 3 -spheres $S U_{\alpha}(2)$ and the integral of $\Omega$ over $S U_{\alpha}(2)$ is by Stokes theorem equal to $1 / 2$ the integral of $\theta$ over $S_{\alpha}^{2}$.

The EXP groupoid construction can be related in a simple way to the use of the central extension $\widehat{\Omega G}$ of the based loop group $\Omega G$ in the construction of the gerbe ove the moduli space $G=\mathcal{A} / \Omega G$ of gauge connections on the circle $S^{1}$. To an element $(s, t)$ in the groupoid $E X P$ one can associate an element $g(s, t) \in \Omega G$ by setting $g(s, t)(\phi)=e^{-\phi s} e^{\phi t}$ for $0 \leq \phi \leq 1$. This is a based closed loop and furthermore

$$
g(s, t)^{-1} s g(s, t)+g(s, t)^{-1} d g(s, t)=t,
$$

so $g(s, t)$ can be interpreted as a gauge transformation taking the constant potential $s$ to the constant potential $t$ on the unit circle.

The curvature of the central extension of EXP is then the pull-back of the left invariant curvature $\frac{k}{2 \pi} \int_{S^{1}}\langle X, d Y\rangle$ on the loop group $\Omega G$, with $X, Y$ in the loop algebra $\Omega \mathfrak{g}$.

\section{REFERENCES}

[1] Alexei Kitaev: Periodic table for topological insulators and superconductors (2009). AIP Conference Proceedings. No.1134. American Institute of Physics , New York, NY, pp. 22 30 .

[2] Shinsei Ryu, Andreas P Schnyder, Akira Furusaki and Andreas W W Ludwig: Topological insulators and superconductors: tenfold way and dimensional hierarchy, New J. Phys. 12 (2010).

[3] Jouko Mickelsson: Chiral anomalies in even and odd dimensions. Comm. Math. Phys. 97 (1985), no. 3, 361 - 370 .

[4] Ludwig Faddeev and Samson Shatashvili: Algebraic and Hamiltonian methods in the theory of nonabelian anomalies. (Russian) Teoret. Mat. Fiz. 60 (1984), no. 2, 206 - 217. 
[5] M.F. Atiyah and I.M. Singer: Dirac operators coupled to vector potentials. Proc. Nat. Acad. Sci. U.S.A. 81 (1984), no. 8, Phys. Sci., 2597 - 2600.

[6] Alan Carey, Jouko Mickelsson and Michael Murray: Index theory, gerbes, and Hamiltonian quantization. Comm. Math. Phys. 183 (1997), no. 3, 707 - 722.

[7] Jouko Mickelsson: Wodzicki residue and anomalies of current algebras. Integrable models and strings (Espoo, 1993), 123 - 135, Lecture Notes in Phys. 436, Springer, Berlin, 1994.

[8] Joakim Arnlind and Jouko Mickelsson: Trace extensions, determinant bundles, and gauge group cocycles. Lett. Math. Phys. 62 (2002), no. 2, 101 - 110.

[9] Christopher Cronström and Jouko Mickelsson: On topological boundary characteristics in nonabelian gauge theory. J. Math. Phys. 24 (1983), no. 10, 2528 - 2531.

[10] Jouko Mickelsson and Stefan Wagner: Third group cohomology and gerbes over Lie groups. J. Geom. Phys. 108 (2016), 49 - 70.

[11] Antti J. Harju and Jouko Mickelsson: Twisted K-theory constructions in the case of a decomposable Dixmier-Douady class. J. K-Theory 14 (2014), no. 2, 247 - 272.

[12] Jouko Mickelsson and S.G. Rajeev: Current algebras in $d+1$ dimensions and determinant bundles over infinite-dimensional Grassmannians. Comm. Math. Phys. 116 (1988), no. 3, $365-400$.

[13] David Shale and W. Forrest Stinespring: Spinor representations of infinite orthogonal groups. Journal of Mathematics and Mechanics, 14:315 - 322, 1965.

[14] Jouko Mickelsson: Gerbes on Quantum Groups. arXiv:math/0308235.

[15] Michael Murray and Danny Stevenson: The basic bundle gerbe on unitary groups. J. Geom. Phys. 58 (2008), no. 11, 1571 - 1590.

[16] Eckhard Meinrenken: The basic gerbe over a compact simple Lie group. Enseign. Math. (2) 49 (2003), no. 3-4, 307 - 333.

Department of Mathematics and Statistics, University of Helsinki

E-mail address: jouko@kth.se 